\title{
COMUNICACIONES
}

\section{Pediculosis y otras ectoparasitosis en una población infantil urbana del nordeste argentino}

\author{
ALICIA M. F. MILANO*, ELENA B. OSCHEROV* y ADRIANA Z. LEGAL*

\begin{abstract}
PEDICULOSIS AND OTHER PARASITOSIS IN A NORTHEAST
\end{abstract} \\ ARGENTINE CHILDREN POPULATION
}

The aim of the present investigation was to evaluate infant pediculosis and other ectoparasitosis in susceptible hosts, presents in the domestic environment of Santa Ana de los Guácaras town, in Corrientes, Argentina. Forty four houses were polled. One hundred and forty eight children between 1 and 14 years-old and eighty domestic animals were parasitological evaluated (54 dogs, 21 cats, three rabbits and two pigs). A range degree of contact between children and animals was established. Direct observation of the environment and the application of semi structured polls were performed to collect the data. The global frequency of infant ectoparasitosis was 58.8\% (87/148). Pediculus capitis was the most frequent ectoparasite, present in $56.8 \%$ of the population, Tunga penetrans in $12.2 \%$ and Pulex irritans in 4.3\%. Two children infested with Ctenocephalides felis and two with Rhipicephalus sanguineus were observed. The animals presented infestation with $\boldsymbol{R}$. sanguineus, $\boldsymbol{C}$. felis, Amblyomma tigrinum, T. penetrans and P. irritans. All the children with lice presented confirmed active pediculosis. Same fleas and ticks species infested human and domestics animals to prove the antropozoonotic character of these arthropods. The infestation was verified in scholar an in less than five years-old, it's necessary to develop control strategies in a short term.

Key words: Pediculosis, Fleas, Ticks, Ectoparasitosis, Environment.

\section{INTRODUCCIÓN}

Los estadios adultos o juveniles de algunos artrópodos pueden parasitar a una amplia gama de mamíferos, entre ellos el hombre, de forma permanente o transitoria.

Entre los parásitos permanentes se destaca el piojo de la cabeza Pediculus capitis (Insecta: Phthiraptera), de hábitos hematófagos que desarrolla todo su ciclo de vida sobre el hospedador. Es el agente etiológico de la pediculosis, de distribución mundial, con prevalencias en el Viejo Mundo que varían entre 6,8\% en Turquía ${ }^{1}$ y 56,7\% en Israel $^{2}$.
En el Nuevo Mundo los porcentajes de infestación en escolares norteamericanos se incrementan año a año ${ }^{3}$. En Latinoamérica los estudios sobre prevalencia son escasos; se informó $26,0 \%$ en Chile, $100,0 \%$ en Perú y 96,0\% en Panamát-6. En Argentina la pediculosis constituye una de las enfermedades más comunes en la infancia, principalmente entre los niños en edad escolar. Los valores de prevalencia son elevados y varían entre $38,1 \%$ y $62,0 \%{ }^{3,7,8}$.

Entre los ectoparásitos temporarios, se encuentran los agentes etiológicos de la pulicosis, infestación que se refiere a la acción hematofágica

\footnotetext{
* Asignatura Biología de los Parásitos. Departamento de Biología. Facultad de Ciencias Exactas y Naturales y Agrimensura. Universidad Nacional del Nordeste. Av. Libertad 5470. 3400 Corrientes. Argentina.
} 
de las pulgas (Insecta: Siphonaptera) sobre el hombre. De distribución cosmopolita, se hallan presentes especialmente en zonas templadas. Todas las especies de pulgas pueden infestar a humanos como hospedadores alternativos. Entre otras: Pulex irritans (pulga del hombre), Ctenocephalides canis (pulga del perro), C. felis (pulga del gato) y Tunga penetrans (nigua, pique o pulga de la arena). Las tres primeras, además del prurito e irritación que provocan, son hospedadores intermediarios de Dipylidium caninum y D. felis, tenias de importancia zoonótica. $T$. penetrans es frecuente en zonas cálidas y de suelos arenosos; la lesión causada por el ingreso y desarrollo de la hembra fecundada puede sobreinfectarse y en casos extremos llegar a la gangrena ${ }^{9}$.

El ciclo biológico de las pulgas incluye periodos que se cumplen en el ambiente, donde se refugian y proliferan, por lo que son consideradas parasitosis ambientales ${ }^{10}$. Tienen amplitud en la elección del hospedador y se alimentan de la sangre de los animales y del hombre, por lo tanto, su presencia está relacionada con la de los animales que comparten la vivienda humana.

Por otra parte se reconoce el papel antropozoonótico de las garrapatas (Arachnida: Parasitiformes) como parásitos de los animales y humanos y como agentes transmisores de patógenos que comprometen el estado de salud de sus hospedadores ${ }^{11}$.

El objetivo del presente trabajo fue evaluar la pediculosis infantil y otras ectoparasitosis en los hospedadores susceptibles, presentes en el ámbito doméstico.

\section{MATERIAL Y MÉTODO}

Área de estudio: La investigación se llevó a cabo entre marzo y diciembre de 2005, en Santa Ana de los Guácaras, área urbana situada en el Departamento San Cosme (27 $27^{\prime}$ S y $58^{\circ} 45^{\prime}$ $\mathrm{W})$, distante $12 \mathrm{Km}$ de la capital de la provincia de Corrientes, Argentina. En la región existe una escasa variación anual entre las temperaturas estivales e invernales lo que determina un clima mesotermal y el régimen pluvial es de aproximadamente $1.500 \mathrm{~mm}$ anuales. Cuenta con 870 habitantes permanentes distribuidos en 273 viviendas.
Encuesta parasitológica y ambiental: El contacto con la población se realizó desde la Institución Escolar N ${ }^{\circ} 105$ Pedro Matoso, donde se informó y explicó sobre el trabajo de investigación, a los directivos, docentes, alumnos y tutores a fin de generar interés e inducir a la participación. Se entregó a los tutores un formulario sencillo solicitando autorización por escrito para visitar sus viviendas y el consentimiento para que sus hijos participen en el estudio. Se incluyeron todas las unidades domésticas de cuyos moradores se obtuvieron respuestas positivas.

Se analizaron por observación directa, durante tres minutos, todos los niños que tenían entre 1 y 14 años de edad, residentes en las viviendas visitadas $^{8}$. La evaluación de ectoparasitosis fue de carácter cualitativa, definiendo presencia o ausencia de algún estadio parasitario. Se definió como pediculosis activa a la presencia de al menos una forma móvil de $P$. capitis $^{12}$. Como variables biológicas se consideraron la edad y el sexo. Las variables ambientales fueron los aspectos sanitarios relacionados con las características de la vivienda donde residían los niños, la posesión de mascotas y el grado de contacto entre los animales y los niños.

La toma de datos ambientales se realizó a través de observación directa del entorno y de la aplicación de encuestas semiestructuradas a la persona a cargo del hogar.

Se registró la presencia y número de animales en cada vivienda. El grado de contacto con los animales se definió como alto cuando la mascota compartía la cama con el niño, medio cuando compartía el dormitorio y bajo cuando la mascota permanecía en zonas externas a los dormitorios.

La evaluación de las ectoparasitosis animales se efectuó del mismo modo que en los niños.

Todos los ectoparásitos fueron extraídos manualmente y conservados en alcohol $70 \%$. Se observó cada uno de los ejemplares bajo lupa estereoscópica Leica Zoom 2000. La determinación taxonómica se realizó utilizando claves específicas ${ }^{13,14}$. Se identificó el estado de desarrollo de los individuos capturados.

Los estudios realizados se ajustaron a las condiciones establecidas por la Declaración Universal de los Derechos Humanos de 1948, las normas éticas instituidas por el Código de Nüremberg de 1947, la Declaración de Helsinki de 1964 y sucesivas enmiendas y a lo normado 
por la Ley Nacional 25.326 de protección de datos de las personas ${ }^{15}$.

El análisis estadístico se realizó con los programas estadísticos Epi-info 6.04 y Epidat 3.1. Se utilizó chi cuadrado $\left(\chi^{2}\right)$ para comparar la prevalencia de acuerdo a la edad y sexo. El intervalo de confianza (IC) fue $95 \%$.

\section{RESULTADOS}

Se encuestaron 44 hogares. Se evaluaron examinaron 148 niños y 80 animales domésticos (54 perros, 21 gatos, tres conejos y dos cerdos).

La frecuencia global de ectoparasitosis infantil fue $58,8 \%$ (87/148). El ectoparásito más frecuente fue $P$. capitis, presente en el $56,8 \%$ de los niños (84/148) seguido por $T$. penetrans en el $12,2 \%$ (18/148) y $P$. irritans en el 4,3\% (5/148). Además se observaron dos niños infestados con $C$. felis y dos con Rhipicephalus sanguineus.

Los valores de prevalencia de $P$. capitis de acuerdo al sexo y la edad, se aprecian en la Tabla 1. No se observó diferencia estadísticamente significativa entre los sexos y entre las edades, si bien en las niñas la prevalencia fue ligeramente superior $\left(\chi^{2}=1,54\right.$; IC 95\%; $\mathrm{p}=0,22$ y $\chi^{2}=8,32$; IC $95 \% ; p=0,08$, respectivamente). La franja etaria más afectada fue 6 a 8 años, tanto en las niñas como en los niños. Después del pico de parasitosis, la prevalencia general disminuye. En el caso de las mujeres, la prevalencia disminuye a partir del valor máximo, sin embargo, en los varones se observa un nuevo aumento luego del pico máximo.

El total de los niños parasitados presentó pediculosis activa confirmada. Se colectaron 164 piojos, de los cuales $54(32,92 \%)$ eran ninfas, 53 $(32,31 \%)$ machos y $29(17,68 \%)$ hembras, mientras que la proporción restante $(17,07 \%)$ estuvo representada por huevos.

Las localizaciones más frecuentes de las lesiones producidas por $T$. penetrans fueron los pies y las manos.

Se observaron ninfas de $R$. sanguineus en las paredes de tres viviendas. Los encuestados se refirieron a la posibilidad de ser atacados por las garrapatas del perro; en solo uno de los niños se registró la infestación, identificándose un macho de $R$. sanguineus.

El 84,1\% (37/44) de las unidades domésticas visitadas poseía uno o más animales. Se evaluaron 54 perros, de los cuales $24(44,4 \%)$ estaban parasitados con $R$. sanguineus, $11(20,4 \%)$ con C. felis, cinco con Amblyomma tigrinum, tres $(5,55 \%)$ con $T$. penetrans y uno de ellos $(1,85 \%)$ presentaba infestación con $P$. irritans. En cuanto a los gatos evaluados $(n=21)$, en seis de ellos $(28,57 \%)$ se comprobó infestación con C. felis, en uno con $R$. sanguineus y en uno se identificó a A. tigrinum. En tres de los tres conejos evaluados se comprobó la infestación con $R$. sanguineus. En uno de los dos cerdos evaluados se observó infestación con $T$. penetrans.

En la Tabla 2 se aprecia la frecuencia de infestación de los niños por pulgas y garrapatas en relación al grado de contacto con los animales domésticos.

En las viviendas que no poseían animales domésticos $(n=7)$ se evaluaron 20 niños, en los que se comprobó sólo infestación por piojos, no registrándose la presencia de pulgas ni garrapatas.

Del análisis de la encuesta surge que sólo el $18,51 \%(\mathrm{n}=10)$ de los perros recibió tratamiento para los ectoparásitos al menos una vez desde la posesión. El resto de los animales domésticos nunca fueron tratados para estas patologías.

Las viviendas analizadas eran similares en

Tabla 1. Prevalencia de Pediculus capitis de acuerdo con el sexo y la edad en niños entre 1 y 14 años. Santa Ana de los Guácaras, Corrientes, Argentina. 2005

\begin{tabular}{lccccccccc}
\hline Edad(años) & n & $\begin{array}{c}\text { Total } \\
\text { Parasitados }\end{array}$ & $\%$ & n & $\begin{array}{c}\text { Femenino } \\
\text { Parasitados }\end{array}$ & $\%$ & \multicolumn{2}{c}{$\begin{array}{c}\text { Masculino } \\
\text { Parasitados }\end{array}$} & \% \\
\hline $1-2$ & 15 & 8 & 53,3 & 7 & 4 & 57,1 & 8 & 4 & 50,0 \\
$3-5$ & 33 & 20 & 60,6 & 17 & 12 & 70,6 & 16 & 8 & 50,0 \\
$6-8$ & 28 & 22 & 78,6 & 15 & 12 & 80,0 & 13 & 10 & 76,9 \\
$9-11$ & 43 & 20 & 46,5 & 24 & 13 & 54,2 & 19 & 7 & 36,8 \\
$12-14$ & 29 & 14 & 48,3 & 15 & 7 & 46,7 & 14 & 7 & 50,0 \\
Total & 148 & 84 & 56,8 & 78 & 48 & 61,5 & 70 & 36 & 51,4 \\
\hline
\end{tabular}


Tabla 2. Frecuencia de infestación infantil con pulgas y garrapatas en relación al grado de contacto con animales domésticos. Santa Ana de los Guácaras, Corrientes, Argentina. 2005

\begin{tabular}{lrccrr}
\hline Grado de contacto & $\mathbf{n}$ & \multicolumn{4}{c}{ Ectoparasitosis (\%) } \\
& & T. penetrans & $\boldsymbol{P}$. irritans & $\boldsymbol{C}$. felis & $\boldsymbol{R}$. sanguineus \\
\hline Alto & 32 & $3(9,4)$ & $3(9,4)$ & - & \\
Medio & 60 & $3(5,0)$ & $1(1,7)$ & $1(1,7)$ & $2(3,3)$ \\
Bajo & 36 & $12(33,3)$ & $1(2,8)$ & $1(2,8)$ & - \\
Total & 128 & $18(14,1)$ & $5(3,9)$ & $2(1,6)$ & $2(1,6)$ \\
\hline
\end{tabular}

sus características de construcción, todas eran de material con techo de chapa. En todas se observó la presencia de áreas de tierra o arena, conformando patios y jardines.

El tratamiento del ambiente está orientado sólo a la eliminación de T. penetrans, mediante el riego de pisos y patios con algún desinfectante mientras que no se uitiliza ningún producto específico para eliminar otras pulgas y las garrapatas.

\section{DISCUSIÓN}

La prevalencia de pediculosis obtenida en este trabajo fue superior a la registrada en otras provincias de Argentina ${ }^{3,7,8}$. Se verificó mayor prevalencia en las niñas, en coincidencia con lo verificado en La Rioja y La Plata ${ }^{3,8}$.

La franja etaria más afectada fue de 6 a 8 años, tanto en las niñas como en los niños, por lo que ésta sería la edad más vulnerable. Después del pico de parasitosis, la prevalencia disminuye pero manteniendo siempre valores altos, por lo que el comportamiento sería diferente al registrado en otras localidades de Argentina ${ }^{8}$.

En la presente investigación se comprobó que todos los niños con piojos presentaron formas móviles. Teniendo en cuenta que los niños con pediculosis activa confirmada son verdaderos diseminadores de piojos a la población $\mathrm{sana}^{8}$, una estricta vigilancia con el fin de evitar nuevas colonizaciones es necesaria.

Si bien el tratamiento químico es el recomendado en los casos de pediculosis activa, se observa en las poblaciones actuales de $P$. capitis resistencia a los pediculicidas tradicionales utilizados hasta el momento ${ }^{16}$, por lo tanto, es importante promover acciones de tratamiento ligadas a la eliminación de todos los estadios, a través de métodos mecánicos como lo es el uso del peine fino. Tanto la acción mecánica como química, antes expuesta constituyen medidas de control y prevención elementales pero efectivas y económicas.

En esta investigación se destaca el carácter antropozoonótico de las pulgas y garrapatas al comprobar la infestación humana y de los animales de compañía con las mismas especies de éstos artrópodos. Asimismo se observa una relación directa entre la ausencia de animales domésticos y las infestaciones infantiles por pulgas y garrapatas.

En una elevada proporción de las viviendas se registró la presencia de animales de compañía, en los que se comprobó la infestación con pulgas y garrapatas. Este hecho sumado al grado de contacto mantenido con los niños, explican la interacción de los ectoparásitos con ambos hospedadores.

La infestación por pulgas, tanto humanas como de los animales domésticos, revela deficiencias en el saneamiento ambiental dentro las viviendas, ya que su prevención y control se basan en la higiene del ambiente, tanto mecánica como química?.

En este sentido, el mantenimiento de la tungiasis en el área de estudio involucra aspectos ambientales y culturales ${ }^{17}$. Por un lado, el suelo arenoso característico del área de estudio y el clima cálido favorecen el desarrollo de esta pulga. Por otro lado, las infestaciones en los animales y humanos son toleradas por los pobladores ya que el papel de las pulgas como parásitos es minimizado, hecho que constituye un obstáculo al momento de adoptar las medidas adecuadas de control ${ }^{18}$.

Las localizaciones mas frecuentes de las lesiones producidas por $T$. penetrans fueron los pies y las manos, acorde con investigaciones llevadas a cabo en Argentina ${ }^{19}$.

En cuanto a la presencia de $P$. irritans en uno 
de los perros analizados, existe coincidencia con hallazgos efectuados en Perú ${ }^{20}$.

$\mathrm{Al}$ igual que en trabajos realizados en Brasil y Argentina, se encontró a $R$. sanguineus parasitando no solamente perros sino también gatos y conejos. Asimismo se confirma la importancia de esta garrapata en salud pública, al comprobar la infestación humana ${ }^{11,21}$.

A. tigrinum, por su ciclo biológico, es un indicador de ambientes frecuentados por roedores. Por lo tanto su presencia sugiere la interacción, a través del ambiente, de dichos animales sinantrópicos con los animales domésticos ${ }^{22}$.

La presencia de los ectoparásitos hematófagos comprobados en este trabajo, no solamente en niños en edad escolar sino también en menores de cinco años, demuestra la necesidad de desarrollar estrategias de control a corto plazo, que incluyan la eliminación de los agentes parásitos, el saneamiento ambiental y la educación sanitaria tendiente a adoptar medidas de control y prevención.

\section{RESUMEN}

El objetivo del presente trabajo fue evaluar la pediculosis infantil y otras ectoparasitosis en los hospedadores susceptibles, presentes en el ámbito doméstico de Santa Ana de los Guácaras, Corrientes, Argentina. Se encuestaron 44 hogares. Se evaluaron parasitológicamente 148 niños entre 1 y 14 años de edad y 80 animales domésticos (54 perros, 21 gatos, tres conejos y dos cerdos). Se estableció el grado de contacto de los niños con los animales. La toma de datos ambientales se realizó a través de observación directa del entorno y de la aplicación de encuestas semiestructuradas. La frecuencia global de ectoparasitosis infantil fue 58,8\% (87/148). El ectoparásito mas frecuente fue Pediculus capitis, presente en el 56,8\% de los niños seguido por Tunga penetrans en el $12,2 \%$ y Pulex irritans en el 4,3\%. Se observaron dos niños infestados con Ctenocephalides felis y dos con Rhipicephalus sanguineus. Los animales presentaron infestación con $R$. sanguineus, C. felis, Amblyomma tigrinum, $T$. penetrans y $P$. irritans. Todos los niños con piojos presentaron pediculosis activa confirmada. Se destaca el carácter antropozoonótico de las pulgas y garrapatas al comprobar la infestación humana y de los animales de compañía con las mismas especies de éstos artrópodos. Los ectoparásitos hematófagos hallados, no solamente en niños en edad escolar sino también en menores de cinco años, demuestra la necesidad de desarrollar estrategias de control a corto plazo.

\section{REFERENCIAS}

1.- KOKTURK A, BAZ K, BUGDAYCI R, et al. The prevalence of pediculosis capitis in schoolchildren in Mersin, Turkey. Int J Dermatol 2003; 42: 694-8.

2.- MUMCUOGLU K, FRIGER M, IOFFE-USPENSKY I, et al. Louse comb versus direct visual examination for the diagnosis of Head Louse Infestations. Pediat Dermatol 2001; 18: 9-12.

3.- VILLALOBOS C, RANALLETTA M, SARANDÓN R, GONZÁLEZ A. La pediculosis de ayer y de hoy. Un estudio epidemiológico sobre la infestación de Pediculus capitis en niños de La Plata, Buenos Aires, Argentina. Entomol Vect 2003; 10 (4): 567-77.

4.- SCHENONE H, FALAHA F, VILLARUEL F, ROJAS A, et al. Infestation by Pediculus capitis in Santiago, Chile. Bol Chil Parasitol 1973; 28: 31-4.

5.- MEINKING T L, TAPLIN D. Comparative Efficacy of treatment for Pediculus capitis Infestations. Arch Dermol 1986; 122 (3): 267.

6.- TAPLIN D, MEINKING T L. Pediculosis Infestations. Pediat Dermatol 1995; 7: 1480-515.

7.- CASTRO D, ABRAMOVICH AH, CICCHINO A C, et al. Prevalencia y estacionalidad de la Pediculosis capitis en la población infanto-juvenil de la región sanitari, Buenos Aires, Argentina. Rev Saúde Publica 1994; 28 (4): 295-9.

8.- CATALÁ S, CARRIZO L, CÓRDOBA M, KHAIRALLA R, et al. Prevalence and parasitism intensity by Pediculus humanus capitis in six to eleven-year-old schoolchildren. Rev Soc Bras Med Trop 2004; 37 (6): 499-501.

9.- LARESCHI M, GONZÁLEZ A, VILLALOBOS C. Siphonaptera-Pulgas. En: Artrópodos de Interés Médico en Argentina. Serie Enfermedades Transmisibles. Fundación Mundo Sano, Ciudad de Buenos Aires, Argentina. Publicación Monográfica 2005; 6: 85-9.

10.- ATÍAS A. Parasitología Clínica. Publ Téc Mediterráneo. Santiago, Chile 1991; 618 p.

11.- NAVA S, GUGLIELMONE A A. Las garrapatas (Acari: Argasidae, Ixodidae) de la Argentina: distribución, hospedadores e importancia sanitaria. $1^{\text {a }}$ Jornada Nacional de Ectoparasitología Veterinaria. Corrientes, Argentina 2006; 13-4.

12.- WILLIAMS L K, REACHER A, MAC KENZIE W R, et al. Lice, nits and school policy. Pediatrics 2001; 107: 1011-5.

13.- LAPAGE G. 1983. Parasitología Veterinaria. Cia Ed Continental, S.A. de C.V. México. 790 pp.

14.- SOULSBY E J L. Parasitología y enfermedades parasitarias en los animales domésticos. Nueva Ed 
Interamericana. México. 1990; 823 pp.

15.- Regulación Jurídica de las Biotecnologías. Disponible en: www.biotech.bioetica.org/bio-etica.

16.- PICOLLO M I, VASSENA C V, MOUGABRE CUETO $\mathrm{GA}$, et al. Resistance to insecticides and effect of synergists on permethrin toxicity in Pediculus capitis (Anoplura: Pediculiidae) from Buenos Aires. J Med Entomol 2001; 37: 721-5.

17.- OSCHEROV E B, MILANO A M F. XVII Congreso Latinoamericano de Parasitología. Mar del Plata, Argentina. Parasitol Latinoam 2005; 60 (2): 160-1.

18.- BAR A R, OSCHEROV E B, MOREIRO A C, MILANO A M F. Representaciones sobre las enfermedades parasitarias en Santa Ana de los Guácaras (Corrientes). Jornadas Comunicaciones Científicas y Tecnológicas. Universidad Nacional del Nordeste. Resistencia, Chaco 2006. Disponible en http: www.unne.edu.ar/cyt/2006.

19.- COSCARÓN M P, GONZÁLEZ A, DE VILLALOBOS C, RANALLETTA M A. Aportes al conocimiento de Tunga penetrans (Linné, 1758) (Siphonaptera): su binomía, clínica, tratamiento y prevención en la República Argentina. En: Actualizaciones en artropodología sanitaria argentina. Serie Enfermedades Transmisibles. Fundación Mundo Sano, Ciudad de
Buenos Aires, Argentina. Publicación Monográfica 2002; 2: 249-52.

20.- ESTARES L P, CHÁVES A, CASAS E. Prevalencia de ectoparásitos de Canis familiares en los distritos de San Juan de Lurigancho, San Martín de Porres, Comas e Independencia de Lima Metropolitana. Rev Inv Vet Perú 1999; 10 (2):

21.- DANTAS-TORRES F, AGUIAR FIGUEREDO L, BRANDÃO FILHO S P. Rhipicephalus sanguineus (Acari: Ixodidae), the brown dog tick, parasitizing humans in Brazil. Rev Soc Bras Med Trop 2006; 39 (1): 64-7.

22.- NAVA S, CAPARROS J A, MANGOLD A J, GUGLIELMONE A A. Ticks (Acari: Ixodida: Argasidae, Ixodidae) infesting humans in northwestern Cordoba province, Argentina. Medicina (Buenos Aires) 2006; 66: 225-8

Agradecimientos: Las autoras agradecen a la Lic. Miryam Damborsky por la lectura crítica del trabajo. Proyecto de Investigación (PI 012/04) subsidiado por la Secretaría General de Ciencia y Técnica, Universidad Nacional del Nordeste.

Correspondencia a:

e-mail:milano@exa.unne.edu.ar 